\title{
Ultrasound-Guided IV Placement by Emergency Department Nurses versus Hospital IV Insertion Team- A Retrospective Study of Time to Successful Insertion
}

\author{
Nicholaus Josey, Virginia LaBond*, Roya Caloia, Jennifer Hella and Kimberly Barber \\ Department of Emergency Medicine, Ascension Genesys Hospital, USA \\ *Corresponding author: Virginia LaBond, Department of Emergency Medicine, Ascension Genesys Hospital, USA
}

\begin{tabular}{l}
\hline ARTICLE INFO \\
Received: 慧 January 18, 2020 \\
Published: 慧 February 18, 2020 \\
\hline
\end{tabular}

Citation: Nicholaus J, Virginia LaB, Roya C, Jennifer $\mathrm{H}$ and Kimberly B. UltrasoundGuided IV Placement by Emergency Department Nurses versus Hospital IV Insertion Team- A Retrospective Study of Time to Successful Insertion. Biomed J Sci \& Tech Res 25(4)-2020. BJSTR. MS.ID.004235.

\section{ABSTRACT}

Background: IV access in the emergency and acute care settings can often present as a challenge for patient care employees who are tasked with the essential task of placing an IV into a patient's vein. This study compares time to IV placement by an IV Resource Insertion team compared to trained ER nurses in the skill of ultrasound guided.

Methods: This was a single site retrospective study which looked at the time to IV placement between a trained team of ER nurses and a formal IV insertion team, both using ultrasound guidance. The primary measure time to IV placement; age, gender, comorbidities and signs of infection were also reported.

Results: Thirty patients were chosen for each group. There was a statistically significant difference in times to IV placement between the ultrasound guided IV ER nurse team and the formal IV insertion team, with shorter times to IV insertion by the trained ER nurse team.

Conclusion: This study lends credibility to the notion that Emergency Department personal should be encouraged to learn and utilize US guided IV insertion to better improve patient waiting times for things such as lab results, IV fluids and antibiotics. This will improve patient experience and care. It will also help to decompress resource stressed health systems.

Abbreviations: US: Ultrasound; HTN: Hypertension; DM: Diabetes; SIRS: Systemic Inflammatory Response Syndromes; BMI: Body Mass Index
What is Already known on this Subject

In ED patients with difficult access, ultrasonography-guided peripheral IVs appear to be an effective alternative to central line placement, which has a relatively high complication rate. The scope of practice of IV nursing teams include a broad range of responsibilities which often prevents rapid attention to difficult access patients in the ED.

\section{What this Study Adds}

This study provides evidence of superior time to IV access when comparing IV Ultrasound technique to IV resource teams providing hospital wide service. Over $50 \%$ of our difficult access patients $(n=60)$ had positive SIRS criteria indicating a need for rapid IV access

\section{Introduction}

Intravenous (IV) access is a necessity of care in the emergency department setting to ensure quality patient care such as hydration, obtaining lab work for diagnosis and the administration of medications [1]. While in most circumstances this is a procedure that is preformed multiple times a day, there are a myriad of reasons which can contribute to delays in IV access being placed effectively and efficiently. The task of establishing IV access usually falls on the 
shoulders of Emergency Department nurses and there are many factors out of their control. Several uncontrollable factors such as poor superficial veins, larger body habitus, severe infections and intravascular volume status (dehydration) can make peripheral IV access all but impossible. Due to this, standard techniques for IV placement may not be feasible. In the setting of sepsis, early antibiotic therapy has been shown to drastically reduce patient's morbidity and mortality during their hospital stay [2]. Many protocols require antibiotics and IV fluids to be given within a certain time frame, and the most current Surviving Sepsis guideline recommendations state that broad spectrum antibiotics should be administered within the first hour of the suspicion of the presence of a septic patient [3].

These cases represent the sickest of the sick patients, many of whom cannot afford to wait long durations for IV access to be placed if initial attempts prove unsuccessful. IV placement in the critically ill patient thus becomes the rate limiting step in the patients care path. There are few options for dealing with this special and challenging population; one of which is consulting an IV resource team. This team is responsible for establishing IV access in difficult patients, often throughout an entire hospital, not just in the Emergency Department [4]. Thus, a consult with this team can lead to a significant delay of patient care in an emergency setting. A delay of 2-3 hours for access in this manner will ultimately delay lab results, imaging and medications administration. The more expedient alternative to this practice is more invasive techniques including central or intraosseous access which present significant risks to the patient $[5,6]$.

With the increasing use of bedside Ultrasound (US) within the Emergency Department, a technique for placement of IV catheters using the ultrasound machine to visualize veins has been developed. Emergency Department staff trained in this technique have improved vascular identification and are not dependent on the timeline of another ancillary service. The nurses utilize the US machine and first identify the vein they would like to place the IV into, next they place the IV into the patients' vein while simultaneously watching their progress on the US monitor to ensure success. This study measured the time from IV order to placement of the IV in patients with difficult IV access. Those patients who received IV team access were compared to those who have had US guided IV (IVUS) placement by their emergency nurse [7]. The study hypothesis was that there would be a significant decrease in time to IV access in the IV team group.

\section{Methods and Materials}

Ethics Committee approval was obtained via IRB on May 14th, 2019. The study was a retrospective design. Data collection of emergency department patients 18 years of age or older, who required advanced IV insertion techniques either by our IV resource team (IVRT) or IVUS trained emergency department nurses (IVUS) resource was obtained for the period of October 1st, 2018, to May 30th, 2019. Study subjects were the individuals preforming the IV insertions grouped into either IV Resource team or the IV Ultrasound guided ER nurses. The cases of this study were the individual patients who underwent the placement of the IVs. IV team records were provided by their service and included patient MRNs (medical record numbers) and through a chart review, data regarding when the IV consult was ordered and when it was completed could be obtained. Additionally, ER nurses charted an IV insertion note which included the time it took to successfully place the IV. The IVRT's time to placement was obtained by looking at the time an order was placed for their service versus when it was completed.

The IVUS team of ER nurses recorded patient MRNs of whom they placed IVs with the ultrasound (US) machine and recorded time to complete attempt as well as how many attempts were required. The potential bias of differential note taking was considered and addressed by a preliminary review of the notes by each group and determined that records were kept objectively and using the same standard rules. The MRNs were also utilized to provide demographic data on all patients including patients age, gender, Body Mass Indexes, presence or absence of Systemic Inflammatory Response Syndromes (SIRS criteria), and comorbidities including diabetes (DM) and hypertension (HTN). These demographics were collected in order to address any differences between the 2 patient groups.

\section{Analysis}

A power calculation was conducted to determine sample size. A minimum of 60 subjects ( 30 per group) was needed to achieve at least $80 \%$ power to detect that these two means $(10 \mathrm{~min}$ vs $30 \mathrm{~min}$; having a relative difference of $60 \%$ ) are significantly different at $\mathrm{p}<0.05$. The primary outcome of measure was time to placement of the IV. The mean difference in time of placement between the two administration groups was compared using a Student's t-Test for Independent groups. A minimum of 60 subjects (30 per group) were needed to achieve at least $80 \%$ power to detect that these two means (10min vs $30 \mathrm{~min}$; having a relative difference of $60 \%$ ) as significantly different at $\mathrm{p}<0.05$. There was no missing data requiring attention or statistical adjustment.

\section{Results}

All data sheets obtained from both groups were complete and without any missing data. The average age of all patient cases was 71.4 years old (SD:68.2). The gender breakdown of the entire study population was $53 \%(n=32)$ of the study population female and $47 \%(n=28)$ were male. The average body mass index (BMI) of all subjects was 30.9 (SD:8.08) with a minimum BMI of 16.4 and a maximum BMI of 50.9. The percent of all patients in the study with HTN and DM was $60 \%(n=36)$ and $28 \%(n=17)$, respectively. Finally, the overall average time to IV placement for all subjects was 123.5 minutes (SD:196.04) with a minimum time of 2 minutes and a maximum time of 800 minutes (Table 1). In the IVUS group, 
the mean age of the patients who received IVs was 80.2 years old (SD:95). The IVUS patients were comprised of $60 \%(n=18)$ females and $40 \%(n=12)$ males. The average BMI of the IVUS group was 32.9 (SD: 7.13) with a minimum of 22.2 and a maximum of 50.9. HTN and DM were present in $46.7 \%(n=14)$ and $20 \%(n=6)$, respectively.

Table 1: IV Ultrasound vs. IV Resource Team - Time to placement.
SIRS signs were present in $56.7 \%(n=17)$ of the IVUS patients. The median time to placement was 5 minutes (range: 2 - 127min) with quartiles $(25$ th $=5,50$ th $=5$, and 75 th $=8.5)$. A 5-minute time was recorded for $63 \%$ of placements in this group.

\begin{tabular}{|c|c|c|c|}
\hline & $\begin{array}{l}\text { Total } \\
\mathrm{N}=60\end{array}$ & $\begin{array}{c}\text { ER IVUS } \\
n=30\end{array}$ & $\begin{array}{l}\text { IVRT } \\
\mathrm{n}=30\end{array}$ \\
\hline Age (mean, SD) & 71.4 (SD 68.2) & 80.2 (SD 95.0) & 62.7 (SD 16.8) \\
\hline $\begin{array}{c}\text { Gender (n, \%) } \\
\text { Male } \\
\text { Female }\end{array}$ & $\begin{array}{l}28(46.7) \\
32(53.3)\end{array}$ & $\begin{array}{l}12(40) \\
18(60)\end{array}$ & $\begin{array}{l}16(53) \\
14(47)\end{array}$ \\
\hline BMI (mean, SD) & 30.9 (SD 8.07) & 32.9 (SD 7.1) & 29 (SD 8.6) \\
\hline HTN & $36(60)$ & $14(46)$ & $22(73.3)$ \\
\hline DM & 17 (28.3) & $6(20)$ & $11(63.3)$ \\
\hline SIRS Signs & $31(51.7)$ & $17(56.7)$ & $14(53.3)$ \\
\hline $\begin{array}{l}\text { Time to placement in minutes } \\
\text { (mean, SD) }\end{array}$ & 132 (SD 196.0) & 10.5 (SD 22.1) & 236.4 (SD 226.5) \\
\hline
\end{tabular}

In the IVRT group the mean age of patients was 62.7 (SD:16.8). The IVRT patients were comprised of $47 \%(n=14)$ females and $53 \%$ $(n=16)$ males. The average BMI of the IVRT group was 29 (SD:8.6) with a minimum of 16.4 and a maximum of 48.9. HTN and DM were present in $73.3 \%(\mathrm{n}=22)$ and $36.7 \%(\mathrm{n}=11)$ of the population, respectively. SIRS signs were present in $46.7 \%(n=14)$ of the IVRT patients. The median time to placement was 164 minutes (range: $21-800 \mathrm{~min})$ with quartiles $(25 \mathrm{th}=65,50 \mathrm{th}=164$, and 75 th $=302)$. Exact time was record for all placements in this group with a mean time of 236.5 minutes (SD: 226.5). Although, the results of this study could have been determined intuitively, we have determined and demonstrated significant superiority of the Emergency Room US guided technique at least in the setting of a hospital with an IV team.

\section{Discussion}

US guided IVs by trained ER personal provided faster times to IV insertion than utilizing the hospitals IVRT due to the unavoidable nature of the IVRT's responsibilities. The IVRT covers all patients in the hospital, are only present in the hospital during certain times of day and days of the week, and if they are occupied placing an IV in a different patient then the next consult to receive an IV would have to wait. The US guided trained ER nurses benefit from the ability to immediately act and proceed to place an IV with the US machine if initial attempts fail. Expanding on this practice in the Emergency Department would benefit all parties by reducing stress on the hospitals IVRT, decreasing waiting times for patients and by allowing more autonomy for Emergency Department personnel. Interestingly, the patients that received IV placement by ultrasound in the Emergency Department on average were older 80.2 years old vs. 62.7 years old, had higher average BMIs and demonstrated more evidence of SIRS signs. This data lends itself to the interpretation that the ER nurses' performance with regards to time was a significant improvement over the standard of care while facing a more challenging patient population. Emergency departments by their very nature are a revolving door of patients, many of whom fail traditional IV access by manual attempts. The ultrasound guided IV insertion modality provides a secondary resource for emergency medicine providers to establish access to provide patients with lifesaving medications, IV fluids and in obtaining laboratory results.

\section{Limitations}

A possible limitation of this study which could have contributed to a difference in IV placement times is that the IVRT and ER nurse IVUS team collected data in different formats, however, our assessment of each form determined that charting was uniform and complete. The generalizability of this study is limited to medical centers with the same system for providing IV access. A hospital wide IV resource team may not be the most efficient method for providing difficult IV access. Further opportunities to expand on this study include future investigation in examining ultrasound guided IV placement by Emergency room nurses compared to the same nurses placing manual IVs to assess whether the standard of care can be improved upon. Additionally, this study only involved a single site and further examination into other various types of emergency departments could further support the data that was discovered from this study. Finally, a study to investigate whether the improved time of IV placement improved morbidity and mortality could further strengthen the clinical based evidence for utilizing this technique.

\section{Conclusion}

This study supports the use of ultrasound guided IV insertion which will become an essential technique in the ED setting. Further 
training should be provided for emergency medical personal to add this skill set to their practice.

\section{Patient and Public Involvement}

No patient involved.

\section{Funding}

There was no provision of funds for this study or conflicts of interest on the part of the investigators.

\section{References}

1. Kornbau C, Lee KC, Hughes GD, Firstenberg MS (2015) Central line complications. Int J Crit Illn Inj Sci 5(3): 170-178.

2. Hallas P, Brabrand M, Folkestad L (2013) Complication with intraosse- ous access: scandinavian users' experience. West J Emerg Med 14(5): 440-443.

3. (2019) Survivingsepsis.org.

4. Larkin Mary RN (1982) NITA: Journal of the National Intravenous Therapy Association 5(2): 80-83.

5. Dargin JM, Rebholz CM, Lowenstein RA, Mitchell PM, Feldman JA (2010) Ultrasounography-guided peripheral intravenous catheter survival in ED patients with difficult access. American Journal of Emergency Medicine 28(1): 1-7.

6. Weiss SL, Fitzgerald JC, Balamuth F, Alpern ER, Lavelle J, et al. (2014) Delayed antimicrobial therapy increases mortality and organ dysfunction duration in pediatric sepsis. Crit Care Med 42(11): 2409-2417.

7. Schoenfeld E, Shokoohi H, Boniface K (2011) Ultrasound-guided peripheral intravenous access in the emergency department: Patient-centered survey. West J Emerg Med 12(4): 475-477.
ISSN: 2574-1241

DOI: $10.26717 / B J S T R .2020 .25 .004235$

Virginia LaBond. Biomed J Sci \& Tech Res

(c) (P) This work is licensed under Creative

Submission Link: https://biomedres.us/submit-manuscript.php Commons Attribution 4.0 License

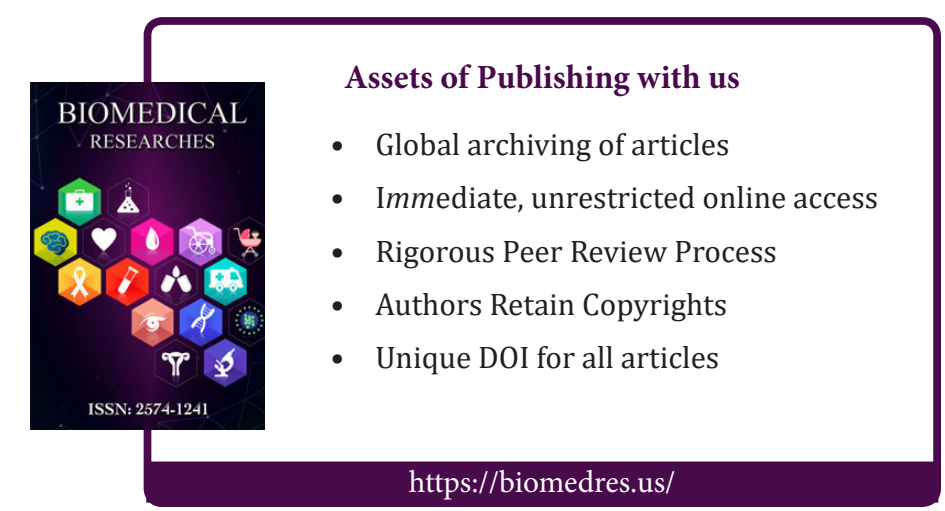

